\title{
Checkpoint kinases and the INO80 nucleosome remodeling complex enhance global chromatin mobility in response to DNA damage
}

\author{
Andrew Seeber, ${ }^{1,2}$ Vincent Dion, ${ }^{1}$ and Susan M. Gasser ${ }^{1,2,3}$ \\ ${ }^{1}$ Friedrich Miescher Institute for Biomedical Research, CH-4058 Basel, Switzerland; ${ }^{2}$ Faculty of Natural Sciences, University \\ of Basel, CH-4056 Basel, Switzerland
}

\begin{abstract}
Double-strand break repair by recombination requires a homology search. In yeast, induced breaks move significantly more than undamaged loci. To examine whether DNA damage provokes an increase in chromatin mobility generally, we tracked undamaged loci under DNA-damaging conditions. We found that the yeast checkpoint factors Mec1, Rad9, and Rad53 are required for genome-wide increases in chromatin mobility, but not the repair protein Rad51. Mec1 activation by targeted Ddc1/Ddc2 enhances chromatin mobility even in the absence of damage. Finally, the INO80 chromatin remodeler is shown to act downstream from Mec1 to increase chromatin mobility, highlighting an additional damage-related role of this nucleosome remodeling complex.
\end{abstract}

[Keywords: DNA damage response; Mec1; chromatin mobility; double-strand breaks; INO80]

Supplemental material is available for this article.

Received May 29, 2013; revised version accepted August 23, 2013.

DNA double-strand breaks (DSBs) can be repaired by either homologous recombination (HR) or nonhomologous end-joining (NHEJ). Whereas NHEJ is the dominant pathway in G1-phase cells and in mammals, the more efficient and preferred pathway of repair in budding yeast is by HR. In the S and G2 phases of the cell cycle, recombinational repair makes use of the undamaged sister chromatid. However, if a sister chromatid is not present or if both sisters have been damaged, a search for an alternative homologous template ensues. This search appears to be rate-limiting for HR (Wilson et al. 1994; Agmon et al. 2013), and its efficiency was predicted to be affected by the mobility of both the break site and the homologous template (Gehlen et al. 2011). This notion is supported by computer simulations, which show that two randomly moving spots confined within a sphere collide more frequently than they would if one were immobile (Gehlen et al. 2011). New data on recombination rates in yeast support these predictions experimentally (Agmon et al. 2013).

Recent studies have also examined the mechanisms that drive chromatin movement of damaged sites (Dion

\footnotetext{
${ }^{3}$ Corresponding author

E-mail susan.gasser@fmi.ch

Article published online ahead of print. Article and publication date are online at http://www.genesdev.org/cgi/doi/10.1101/gad.222992.113.
}

et al. 2012; Krawczyk et al. 2012; Mine-Hattab and Rothstein 2012; Neumann et al. 2012; for review, see Dion and Gasser 2013). The recruitment of repair proteins, such as the strand exchange protein Rad51, enhances the movement of the broken DNA locus, tagged by Rad52-YFP (Dion et al. 2012). By analogy to the bacterial RecA, which contributes to the sequence search in threedimensional (3D) space in vitro (Forget and Kowalczykowski 2012), Rad51 has also been suggested to mechanistically drive homology search (Renkawitz et al. 2013). In eukaryotes, Rad51 recruits the Snf2-type ATPase Rad54, which also contributes to the enhanced mobility of a DSB through an unknown mechanism (Dion et al. 2012).

Alongside these repair proteins, the DNA damage response (DDR) pathway appears to regulate the movement of the DSB. The resection of DNA at a cut site leads to activation of the ATR kinase complex Mec1/Ddc2 and, in turn, the downstream checkpoint kinase Rad53. These kinases regulate a number of processes, including cell cycle transitions, transcriptional programs, and DNA repair. Importantly, both $\mathrm{Mecl}$ and its target protein, Rad9, were needed to increase the mobility of a DSB, whereas Rad53 was not (Dion et al. 2012).

It has remained unresolved whether undamaged chromatin also becomes more mobile in a nucleus that contains DNA damage. One report showed that the induction of a DSB at the MAT locus on chromosome (Chr) III 
in diploid yeast cells led to increased mobility of an undamaged site on the short arm of Chr V (Mine-Hattab and Rothstein 2012). In contrast, Dion et al. (2012) showed that an I-SceI endonuclease-induced DSB in haploid cells did not increase the mobility of an undamaged Chr VI locus, nor did treating cells with the DNA-damaging agent Zeocin at $50 \mu \mathrm{g} / \mathrm{mL}$. The source of this discrepancy was unclear. Factors likely to influence the outcome include (1) the differential regulation of HR in haploid versus diploid yeast cells, (2) the types and levels of damage induced, or (3) the specific genomic context of the locus monitored; e.g., its proximity to a telomere or centromere. Indeed, the nuclear organization of chromosomes and chromosome territories do seem to affect the efficiency of repair by HR (Agmon et al. 2013). In this study, we set out to resolve this discrepancy. We found that checkpoint kinases in yeast induce a genome-wide alteration in chromatin structure, which is manifested as enhanced locus mobility, even in the absence of DNA damage. This increase in mobility appears to be driven by the INO80 nucleosome remodeling complex.

\section{Results}

DNA damage increases global chromatin mobility independently of Rad51

Here we exploited single-particle tracking of fluorescently tagged genomic loci in Saccharomyces cerevisiae to quantify the mobility of the chromatin fiber in vivo. To this end, we recorded 3D image stacks on a spinning-disc confocal microscope every $1.5 \mathrm{sec}$ during $5 \mathrm{~min}$. The images were then deconvolved (Ponti et al. 2007) and projected onto a two-dimensional (2D) plane (Fig. 1A). Using the ImageJ plug-in, spots were tracked with respect to the center of the nucleus (SpotTracker) (Sage et al. 2005), and the $X$ and $Y$ coordinates of the spot as well as the center of the nucleus were determined in each of the 200 images of a typical time-lapse movie. From these values, we calculated the mean-squared displacement (MSD $=\left\langle X_{t}-X_{t+\Delta t}\right\rangle^{2}$, where $X$ is the position of a spot at time $t$ ). From the MSD plot, we derived the radius of constraint ( $\mathrm{Rc}$; the square root of the plateau of the MSD curve multiplied by 5/4) (Meister et al. 2010; Neumann et al. 2012), which is a robust measurement of locus confinement given that thousands of data points were averaged in each graph (Meister et al. 2010).

We tracked genomic loci that were tagged with mCherry-TetR (e.g., the met10 locus on Chr VI) (Fig. 1A) or GFP-LacI (e.g., ATG2 on the long arm of Chr XIV or PES4 and $H X K 1$, both on Chr VI). These haploid cells also express yellow fluorescent protein (YFP) fused to Rad52, which is fully functional for HR (Supplemental Table S1; Lisby et al. 2004). To ensure that we tracked an undamaged locus, timelapse movie data was used only if the genomic tagged locus did not colocalize with Rad52-YFP. Moreover, we confirmed that our imaging regime itself does not induce damage by showing that cells divide with normal kinetics after imaging (Supplemental Fig. S1; Dion et al. 2012).
In line with earlier studies, we measured an Rc of 0.41 $\mu \mathrm{m}$ for the met10 locus in haploid S-phase cells grown on glucose in the absence of damage (Dion et al. 2012; MineHattab and Rothstein 2012). This value indicates that the locus can sample $\sim 9.5 \%$ of the nuclear volume (nvol) (Fig. 1D). We note that movement is higher in G1-phase cells as compared with S-phase cells (Heun et al. 2001) and that the Rc of undamaged loci can range from 0.4 to $0.6 \mu \mathrm{m}$, depending on the chromosomal location of the tagged locus (Table 1; Supplemental Fig. S2; Heun et al. 2001; Gartenberg et al. 2004; Bystricky et al. 2009).

To examine the movement of the undamaged locus in a cell responding to DNA damage, we next exposed cells bearing the tagged met10 locus to increasing doses of Zeocin, a copper-chelated glycopeptide antibiotic that induces both DSBs and single-strand nicks, in the ratio of $\sim$ 1:9 (Povirk 1996; Burger 1998). After $1 \mathrm{~h}$ of treatment with Zeocin, we assessed DDR activation by scoring for phosphorylated forms of H2A and Rad53 (Fig. 1B) and the frequency of Rad52-YFP foci formed (Fig. 1C). Surprisingly, the tagged and undamaged met10 locus exhibited an increase in mobility after Zeocin treatment, increasing with the concentration of Zeocin used and reaching a maximum of nvol searched of $\sim 34 \%$ at $250 \mu \mathrm{g} / \mathrm{mL}$ Zeocin (Fig. 1D). The tracked locus did not colocalize with Rad52-YFP, although each nucleus analyzed had a Rad52-YFP focus elsewhere at both the beginning and end of the movie. In agreement with our previous report, low-level Zeocin $(50 \mu \mathrm{g} / \mathrm{mL})$ did not increase mobility at an undamaged site (Dion et al. 2012), but inducing more damage with higher levels of Zeocin did (Fig. 1C,D).

We then examined whether the increase in chromatin mobility was locus-dependent by scoring the movement of three LacO-tagged loci (PES4, ATG2, and HXK1) on different chromosomes. Each showed an equivalent increase in mobility upon Zeocin treatment, although their chromosomal locations varied significantly, with one being subtelomeric (HXK1) (Supplemental Fig. S2A,B). The undamaged loci tested all showed an increase in mobility, albeit slightly less than that scored at the site of damage (Fig. 1E; Table 1).

To rule out that the increase was due to secondary effects unique to Zeocin, we expressed the restriction enzyme EcoRI in yeast cells bearing the tagged met10 locus. EcoRI induces DSBs that can be monitored through Rad52-YFP foci formation (Fig. 1C). We found that induction of EcoRI also increased the Rc of the undamaged met10 locus from $0.39 \mu \mathrm{m}$ to $0.51 \mu \mathrm{m}$ (from $\sim 9 \%$ to $\sim 18 \%$ nvol) (Fig. 1F). This increase is more modest than that scored at $250 \mu \mathrm{g} / \mathrm{mL}$ Zeocin and correlates with the reduced number of Rad52 foci formed upon EcoRI induction (Fig. 1C).

Given that specialized chromosomal domains (e.g., telomeres or centromeres) have been implicated in both constraining movement (Heun et al. 2001) and limiting recombination (Agmon et al. 2013), we speculated that the general increase in chromatin movement might arise from the release of perinuclear chromosomal anchorage points. To test this, we scored whether the subtelomeric, LacO-tagged locus HXK1 loses its anchorage and moves 
A
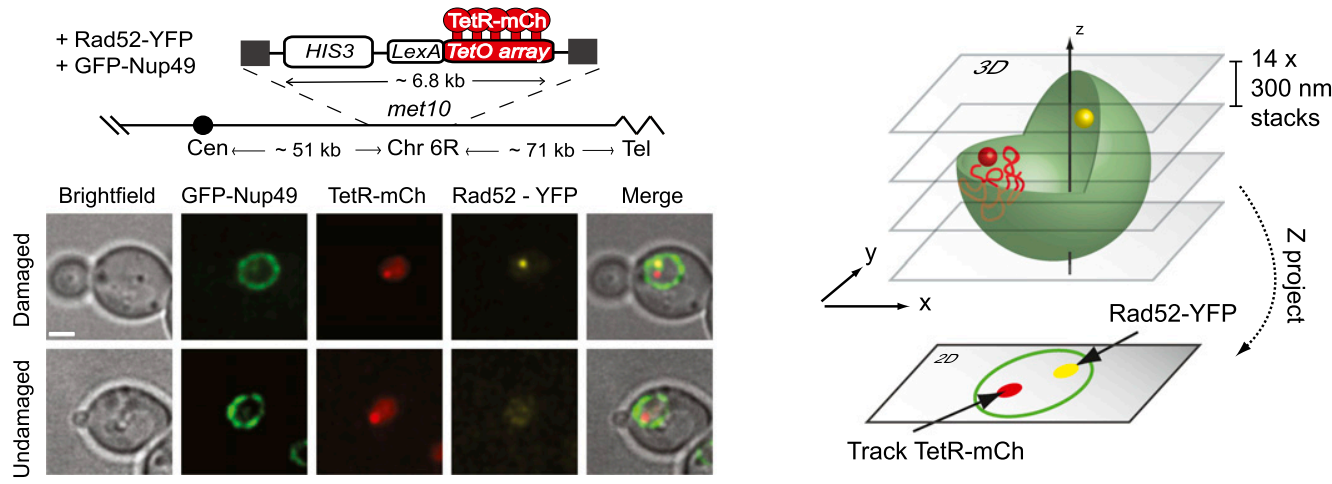

B

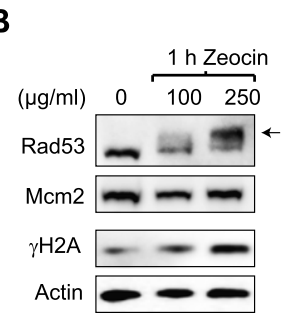

E

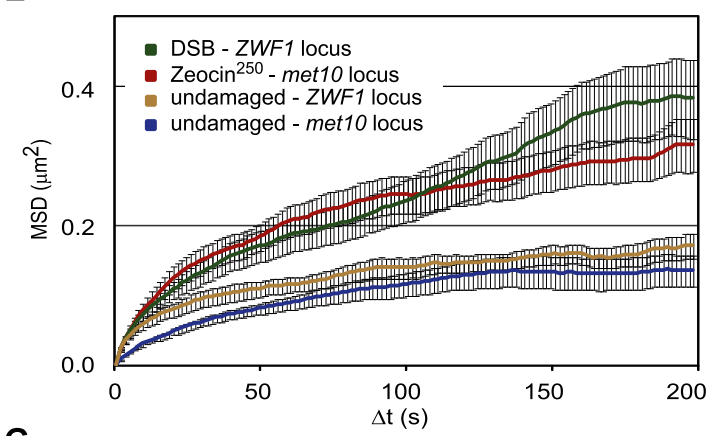

G
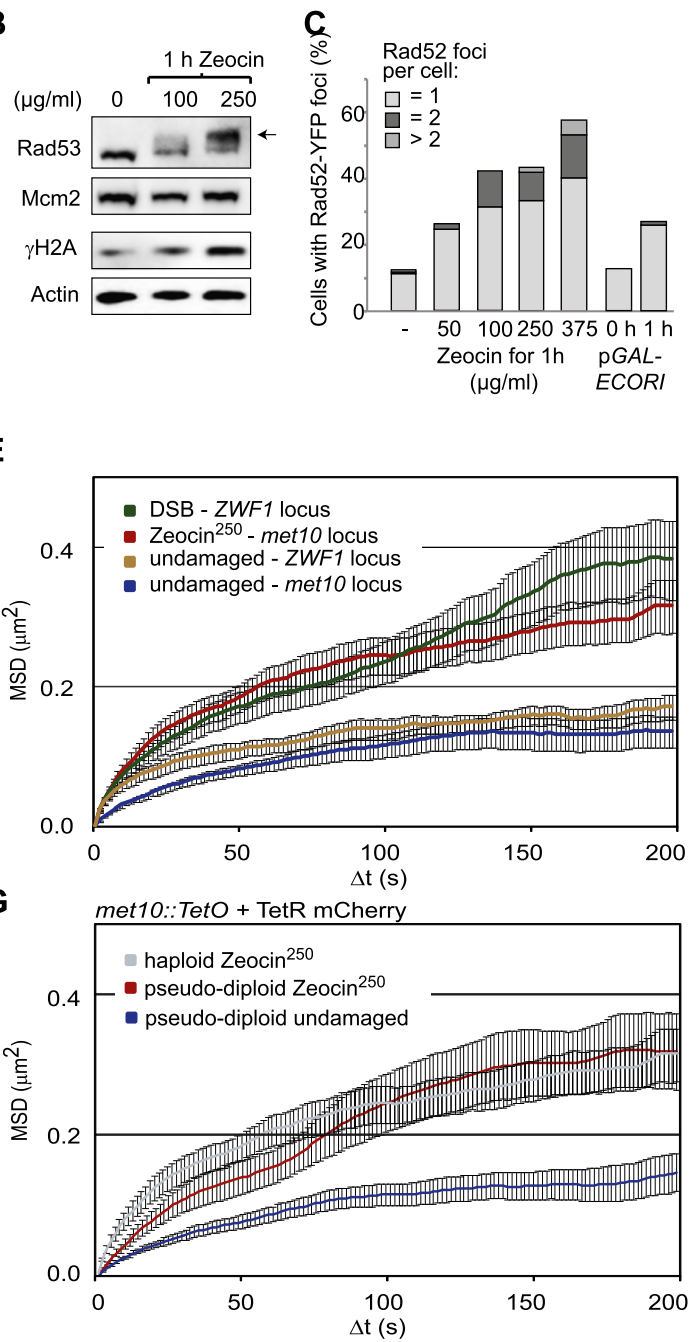

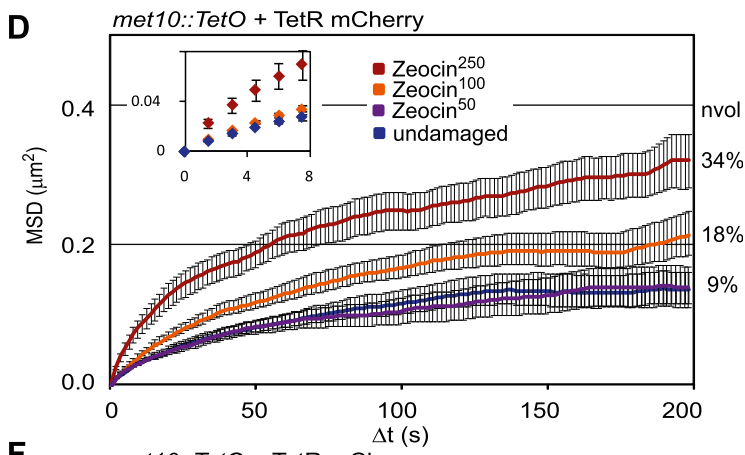

$\mathbf{F}$

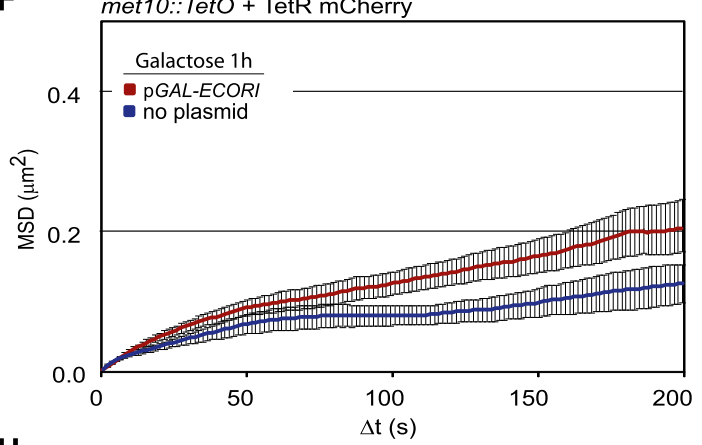

H

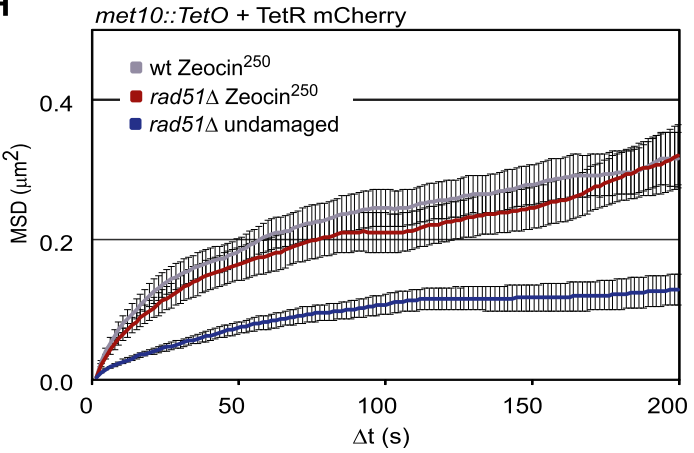

Figure 1. DNA damage causes a global increase in chromatin mobility. (A, top) Schematic of strain GA-6879 showing the tracked met10:: TetO Locus. (Bottom) S-phase cell (GA-6879) after $1 \mathrm{~h}$ of Zeocin or undamaged. Shown is nuclear pore (GFP-Nup49), TetR-mCherry locus, and Rad52-YFP damage focus. Bar, $2 \mu \mathrm{m}$. (Right) Image stack is projected onto a 2D plane for tracking. (B) Western blot of Rad53 and H2A phosphorylation after 1-h Zeocin treatment of GA-6879. Actin and Mcm2 were used as loading controls. (C) Rad52-YFP foci accumulation after 1-h treatment with Zeocin or induction of $p G A L-E C O R I$. Numbers of nuclei scored: 510 (undamaged), 311 (50 $\mu \mathrm{g} / \mathrm{mL} Z \mathrm{Zeocin}), 197$

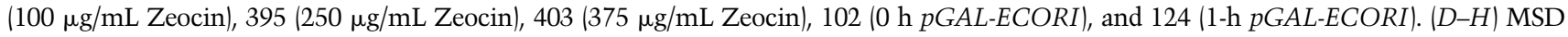
plots of TetO-tagged met10 in S-phase cells. $(D)$ Without damage (blue) or incubated with Zeocin at 50, 100, or $250 \mu \mathrm{g} / \mathrm{mL}$. The inset graph represents the first five time intervals to generate the initial slope. The percentage nuclear volume (nvol) explored is indicated. (E) MSD plot of LacO-tagged ZWF1 (brown) and Rad52-YFP (green) compared with met10::TetO either with $1 \mathrm{~h}$ of Zeocin (red) or without (blue). ZWF1 and I-Scel-induced Rad52-YFP from Dion et al. (2012). (F) MSD plots of met10::TetO after growth in $2 \%$ galactose for $1 \mathrm{~h}$ either with $p G A L$ ECORI (red) or without (blue). (G) MSD plots of met10::TetO during S phase in a pseudodiploid strain (GA-7591) with an extra copy of $M A T \alpha$ either without damage (blue) or after $1 \mathrm{~h}$ of $250 \mu \mathrm{g} / \mathrm{mL}$ Zeocin (red); MSD of met10::TetO in haploid GA-6879 (gray). (H) MSD of met10::TetO in rad51s cells (GA-7550) without damage (blue) or after $1 \mathrm{~h}$ of $250 \mu \mathrm{g} / \mathrm{mL}$ Zeocin (red) or in wild-type cells (GA-6879) in $250 \mu \mathrm{g} / \mathrm{mL}$ Zeocin (light gray). All error bars show the SEM. The numbers of movies tracked and parameters are given in Table 1. 
Table 1. Summary of MSD results presented in this study

\begin{tabular}{|c|c|c|c|c|c|}
\hline Spot tracked & Locus & Treatment & Features & $\mathrm{Rc}$ & $\begin{array}{c}\text { Cell } \\
\text { number }\end{array}$ \\
\hline mCh-TetR & met10::TetO & Undamaged & & $0.41 \mu \mathrm{m} \pm 0.03 \mu \mathrm{m}$ & 20 \\
\hline mCh-TetR & met10::TetO & Zeocin $50 \mu \mathrm{g} / \mathrm{mL}$ & & $0.42 \mu \mathrm{m} \pm 0.04 \mu \mathrm{m}$ & 17 \\
\hline mCh-TetR & met10::TetO & Zeocin $100 \mu \mathrm{g} / \mathrm{mL}$ & & $0.51 \mu \mathrm{m} \pm 0.05 \mu \mathrm{m}$ & 13 \\
\hline mCh-TetR & met10::TetO & Zeocin $250 \mu \mathrm{g} / \mathrm{mL}$ & & $0.63 \mu \mathrm{m} \pm 0.04 \mu \mathrm{m}$ & 28 \\
\hline CFP-LacI & $Z W F 1$ & Undamaged $^{\mathrm{a}}$ & & $0.46 \mu \mathrm{m} \pm 0.02 \mu \mathrm{m}$ & 20 \\
\hline Rad52-YFP & $Z W F 1$ & I-Sce $1^{\mathrm{a}}$ & & $0.7 \mu \mathrm{m} \pm 0.05 \mu \mathrm{m}$ & 17 \\
\hline mCh-TetR & met10::TetO & СССР $40 \mu \mathrm{M}$ & & $0.08 \mu \mathrm{m} \pm 0.007 \mu \mathrm{m}$ & 10 \\
\hline mCh-TetR & met10::TetO & $\begin{array}{l}\text { CССР } 40 \mu \mathrm{M}+\text { Zeocin } \\
250 \mu \mathrm{g} / \mathrm{mL}\end{array}$ & & $0.2 \mu \mathrm{m} \pm 0.06 \mu \mathrm{m}$ & 6 \\
\hline mCh-TetR & met10::TetO & Undamaged & Pseudodiploid & $0.43 \mu \mathrm{m} \pm 0.04 \mu \mathrm{m}$ & 15 \\
\hline mCh-TetR & met10::TetO & Zeocin $250 \mu \mathrm{g} / \mathrm{mL}$ & Pseudodiploid & $0.64 \mu \mathrm{m} \pm 0.05 \mu \mathrm{m}$ & 12 \\
\hline mCh-TetR & met10::TetO & Control $^{\mathrm{a}}$ & No plasmid-gal & $0.39 \mu \mathrm{m} \pm 0.04 \mu \mathrm{m}$ & 12 \\
\hline mCh-TetR & met10::TetO & Induced $E C O-R I$ & $\mathrm{p} G A L-E C O R I$-gal & $0.51 \mu \mathrm{m} \pm 0.05 \mu \mathrm{m}$ & 15 \\
\hline mCh-TetR & met10::TetO & Undamaged & $\operatorname{sml1\Delta }$ & $0.35 \mu \mathrm{m} \pm 0.02 \mu \mathrm{m}$ & 30 \\
\hline mCh-TetR & met10::TetO & Zeocin $250 \mu \mathrm{g} / \mathrm{mL}$ & $\operatorname{sml1\Delta }$ & $0.47 \mu \mathrm{m} \pm 0.04 \mu \mathrm{m}$ & 26 \\
\hline mCh-TetR & met10::TetO & Undamaged & mec1s sml1s & $0.32 \mu \mathrm{m} \pm 0.03 \mu \mathrm{m}$ & 19 \\
\hline mCh-TetR & met10::TetO & Zeocin $250 \mu \mathrm{g} / \mathrm{mL}$ & mec1s sml1s & $0.36 \mu \mathrm{m} \pm 0.04 \mu \mathrm{m}$ & 16 \\
\hline mCh-TetR & met10::TetO & Undamaged & rad $9 \Delta$ & $0.49 \mu \mathrm{m} \pm 0.03 \mu \mathrm{m}$ & 26 \\
\hline mCh-TetR & met10::TetO & Zeocin $250 \mu \mathrm{g} / \mathrm{mL}$ & rad $9 \Delta$ & $0.46 \mu \mathrm{m} \pm 0.03 \mu \mathrm{m}$ & 21 \\
\hline mCh-TetR & met10::TetO & Undamaged & rad53s sml1s & $0.39 \mu \mathrm{m} \pm 0.02 \mu \mathrm{m}$ & 18 \\
\hline mCh-TetR & met10::TetO & Zeocin $250 \mu \mathrm{g} / \mathrm{mL}$ & rad53s sml1s & $0.41 \mu \mathrm{m} \pm 0.04 \mu \mathrm{m}$ & 24 \\
\hline mCh-TetR & met10::TetO & Undamaged & $\operatorname{rad} 51 \Delta$ & $0.40 \mu \mathrm{m} \pm 0.04 \mu \mathrm{m}$ & 21 \\
\hline mCh-TetR & met10::TetO & Zeocin $250 \mu \mathrm{g} / \mathrm{mL}$ & $\operatorname{rad} 51 \Delta$ & $0.63 \mu \mathrm{m} \pm 0.09 \mu \mathrm{m}$ & 13 \\
\hline GFP-LacI & PES4::LacO & GFP-LacI & No plasmid-gal & $0.38 \mu \mathrm{m} \pm 0.04 \mu \mathrm{m}$ & 14 \\
\hline Ddc1/Ddc2-GFP-LacI & PES4::LacO & Gal-DDC1/2-FP-LacI & Ddc1/2-GFP-LacI-induced-gal & $0.5 \mu \mathrm{m} \pm 0.04 \mu \mathrm{m}$ & 16 \\
\hline mCh-TetR & LEU2::TetO & GFP-LacI & No Ddc1/2-gal & $0.27 \mu \mathrm{m} \pm 0.04 \mu \mathrm{m}$ & 11 \\
\hline mCh-TetR & LEU2::TetO & Gal-DDC1/2-GFP-LacI & Ddc1/2-GFP-LacI-induced-gal & $0.41 \mu \mathrm{m} \pm 0.0 \mu \mathrm{m} 4$ & 11 \\
\hline mCh-TetR & LEU2::TetO & $\begin{array}{l}\text { Gal-DDC1/2-LacI } \\
\text { (no LacO) }\end{array}$ & Ddc1/2-GFP-LacI-induced-gal & $0.30 \mu \mathrm{m} \pm 0.03 \mu \mathrm{m}$ & 9 \\
\hline mCh-TetR & met10::TetO & Undamaged & $\operatorname{arp} 8 \Delta$ & $0.42 \mu \mathrm{m} \pm 0.02 \mu \mathrm{m}$ & 14 \\
\hline mCh-TetR & met10::TetO & Zeocin $250 \mu \mathrm{g} / \mathrm{mL}$ & $\operatorname{arp} 8 \Delta$ & $0.43 \mu \mathrm{m} \pm 0.02 \mu \mathrm{m}$ & 13 \\
\hline mCh-TetR & met10::TetO & Undamaged & $\operatorname{arp} 8 \Delta^{+} p A R P 8-U R A 3$ & $0.46 \mu \mathrm{m} \pm 0.04 \mu \mathrm{m}$ & 16 \\
\hline mCh-TetR & met10::TetO & Zeocin $250 \mu \mathrm{g} / \mathrm{mL}$ & $\operatorname{arp} 8 \Delta^{+} p A R P 8-U R A 3$ & $0.63 \mu \mathrm{m} \pm 0.05 \mu \mathrm{m}$ & 20 \\
\hline mCh-TetR & met10::TetO & Undamaged & $\operatorname{arp} 5 \Delta$ & $0.39 \mu \mathrm{m} \pm 0.05 \mu \mathrm{m}$ & 8 \\
\hline mCh-TetR & met10::TetO & Zeocin $250 \mu \mathrm{g} / \mathrm{mL}$ & $\operatorname{arp} 5 \Delta$ & $0.44 \mu \mathrm{m} \pm 0.04 \mu \mathrm{m}$ & 21 \\
\hline GFP-LacI & PES4::LacO & GFP-LacI & $\operatorname{arp} 8 \Delta$ no Ddc1/2-gal & $0.37 \mu \mathrm{m} \pm 0.02 \mu \mathrm{m}$ & 15 \\
\hline Ddc1/Ddc2-GFP-LacI & PES4::LacO & Gal-DDC1/2-GFP-LacI & $\begin{array}{l}\text { arp } 8 \Delta ; \text { Ddc1/2-GFP } \\
\text { LacI-induced-gal }\end{array}$ & $0.41 \mu \mathrm{m} \pm 0.03 \mu \mathrm{m}$ & 12 \\
\hline mCh-TetR & LEU2::TetO & GFP-LacI & $\begin{array}{l}\operatorname{arp} 8 \Delta ; \text { Ddc1/2-GFP- } \\
\text { LacI-induced-gal }\end{array}$ & $0.30 \mu \mathrm{m} \pm 0.02 \mu \mathrm{m}$ & 10 \\
\hline mCh-TetR & LEU2::TetO & $\begin{array}{l}\text { Gal-DDC1/2-LacI } \\
\text { (no LacO) }\end{array}$ & $\begin{array}{l}\text { arp } 8 \Delta ; \text { Ddc } 1 / 2 \text {-GFP- } \\
\text { LacI-induced-gal }\end{array}$ & $0.31 \mu \mathrm{m} \pm 0.02 \mu \mathrm{m}$ & 13 \\
\hline
\end{tabular}

Indicated are the exact Rc values calculated from the indicated number of time-lapse series for the indicated loci. The "Features" column lists relevant phenotype/genotype and cell cycle stage and indicates when galactose (gal) replaced glucose.

${ }^{a}$ Data points from Dion et al. (2012).

away from the nuclear envelope after DNA damage. Although HXK1 shows increased mobility, it does not lose its perinuclear localization upon incubation with Zeocin, suggesting that movement along the nuclear envelope increases (Supplemental Fig. S2D; Supplemental Table S2). This agrees with earlier results showing that yeast telomeres remained associated with the nuclear envelope after single DSB induction (Martin et al. 1999). The observed increase in chromatin mobility in the presence of damage is therefore not a passive event resulting from a loss of telomere anchoring.

Chromatin mobility in general depends on ATP (Heun et al. 2001; Weber et al. 2012), and the ionophore carbonyl cyanide m-chlorophenyl hydrazine (CCCP) was shown to reduce the mobility of chromosomal loci as well as of an excised chromatin ring (Heun et al. 2001; Gartenberg et al. 2004). Consistently, we found that preincubation of cells with $40 \mu \mathrm{M}$ CCCP suppressed the general chromatin mobility induced by DNA damage (Supplemental Fig. S3A; Supplemental Table S2), suggesting a role for an active mechanism in this process.

The HR repair factors Rad51 and Rad54 are needed to increase mobility at the site of damage itself (Dion et al. 2012; Mine-Hattab and Rothstein 2012). We asked whether repair factors are similarly required for the increased mobility scored at undamaged loci, provoked by DNA lesions elsewhere in the genome. This is not the case; the Rc of the met 10 locus started at the same point in rad51s 
and $R A D 51^{+}$cells and increased after incubation with 250 $\mu \mathrm{g} / \mathrm{mL}$ Zeocin to equal degrees, reaching $\sim 34 \%$ of nvol in both of these otherwise isogenic haploid strains (Fig. 1H).

In diploid yeast, Rad51 is hyperactive (Morgan et al. 2002), and it was suggested (Ira and Hastings 2012) that this hyperactivity might be responsible for the enhanced mobility observed at unbroken sites in diploid cells. To test this hypothesis, we integrated an extra copy of MAT bearing the opposite mating type information (MAT $\boldsymbol{\alpha}$ ) into our wild-type haploid strain (MATa). Rad51 is hyperactivated in this pseudodiploid strain, as in diploid yeast (Morgan et al. 2002; Haber 2012). Tracking of the met10 locus yielded an increase in Rc upon treatment with Zeocin (from $0.43 \mu \mathrm{m}$ to $0.65 \mu \mathrm{m}$ ) in the pseudodiploid that was indistinguishable from that in the haploid strain (Fig. 1G). Thus, the increase in chromatin mobility in response to damage is independent of the Rad51 hyperactivity associated with diploid cells and occurs equally in haploid and diploid strains.

The stage of the cell cycle has a clear effect on chromatin mobility, since in S-phase, movement is much decreased compared with G1 (Heun et al. 2001) due to constraint from sister-sister cohesion (Dion et al. 2013). We were unable to test G1-phase cells, since we used Rad52 focus formation as a marker for damage, and G2-phase nuclei often have distorted nuclear shapes that interfere with accurate tracking. We did score for differences in mobility between early and mid-S phase at met10 and found a similar increase in response to damage at both stages (Supplemental Fig. S3B,C; Supplemental Table S2). Given that met10 is early-replicating, it is likely that the tracked loci are replicated and have cohesin loaded (Dion et al. 2013).

\section{The DDR increases global chromatin mobility}

An earlier study showed that loss of Mec1, the homolog of human ATR kinase, compromised the increased mobility scored for an induced DSB, while loss of Rad53 (CHK2) did not (Dion et al. 2012). It was therefore of interest to test whether checkpoint kinase activation was necessary for the increase in chromatin mobility genome-wide. This was scored in a sml1s background because ablation of Smll up-regulates dNTP synthesis and suppresses the

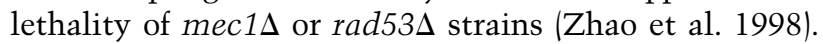
Upon tracking met10, we found that the background chromatin mobility $(\mathrm{Rc}=0.32 \mu \mathrm{m})$ in mec1 $1 \mathrm{sm} l 1 \Delta$ cells was nearly unchanged after $1 \mathrm{~h}$ of exposure to Zeocin $(0.36 \mu \mathrm{m})($ Fig. 2A), and loss of the DDR effector kinase Rad53 completely ablated the damage-induced increase in global chromatin mobility (Fig. 2B). Consistent with earlier observations, sml1 deletion itself has a partial phenotype: The increase in general chromatin mobility after damage in a sml1s strain reaches $0.47 \mu \mathrm{m}$ instead of the $0.65 \mu \mathrm{m}$ of wild-type cells (Fig. 2C). This lower increase in mobility may reflect the fact that sml1s strains have altered dNTP levels, although overexpression of the factor Smll, which down-regulates Rnrl, did not have a similar effect (data not shown). Nonethe-

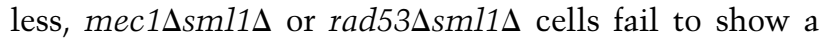

damage-induced, global increase in chromatin mobility, while $s m l 1 \Delta$ cells do.

Next, we tested the role of factors downstream from Mec1. One key target of Mec1 that contributes to both Rad53 activation and the downstream response is the BRCT-containing protein Rad9 (Vialard et al. 1998). Whereas RAD9 deletion alone increased chromatin mobility in the undamaged state, it completely blocked the general chromatin mobility increase induced by Zeocin (Fig. 2D). By Western blot analysis, we confirmed that checkpoint activation was compromised in all of the DDR mutants tested. Interestingly, although mobility did not increase in the rad9 9 mutant, H2A phosphorylation was comparable with that in wild-type cells, indicating that this $\mathrm{Mec} 1$ phosphorylation target $(\gamma \mathrm{H} 2 \mathrm{AX}$ in mammals) does not induce chromatin movement (Fig. 2E), while the DNA checkpoint signaling cascade, which ends with Rad53 activation, does (Fig. 2F). Interestingly, Rad53, unlike Mec1/Ddc2, has a dispersed nuclear localization, allowing it to propagate changes throughout the nucleoplasm (Melo et al. 2001).

\section{Checkpoint activation without DNA damage increases global chromatin mobility}

The question remained whether checkpoint activation is sufficient to enhance chromatin movement in the absence of damage. To examine this, we constructed a strain containing GFP- and LacI-tagged versions of both Ddc1 and Ddc2, whose artificial juxtaposition is sufficient to initiate a damage-independent checkpoint response (Bonilla et al. 2008). Upon expression from a galactoseinducible promoter, these two proteins bound the integrated LacO array at PES4 near MET10 and created a GFP focus that could be tracked (Fig. 3A,B). Upon induction of Ddc1/Ddc2-GFP-LacI, the checkpoint was activated as indicated by H2A phosphorylation (Fig. 3C), and the Rc of the tagged PES4 locus increased from 0.38 $\mu \mathrm{m}$ to $0.5 \mu \mathrm{m}$ (Fig. $3 \mathrm{~B}, \mathrm{D})$.

To see whether checkpoint activation in the absence of DNA damage suffices to trigger the general increase in chromatin mobility, we monitored the LEU2 ::TetO locus in these same conditions. Whereas overexpression of Ddc1/2-GFP-LacI without a LacO array at PES4 did not cause an increase in mobility at LEU2, by targeting Ddc1/ Ddc2 to PES4::LacO, not only did the PES4 locus increase mobility, but also $L E U 2$, albeit to a lower extent (Fig. $3 \mathrm{~B}, \mathrm{E})$. Given that we saw a dose-dependent increase in mobility with Zeocin, we assume that this more modest increase reflects the efficiency of checkpoint activation. We conclude that DNA damage checkpoint activation is sufficient, even in the absence of DNA damage, to increase both local and global chromatin mobility.

\section{Intact INO80 remodeler complex is required} to increase chromatin mobility in trans

Several chromatin remodeling enzymes are targets of Mec1 and the checkpoint response (Morrison et al. 2007; Smolka et al. 2007). Since INO80 is known to increase the mobility of a locus to which it is targeted (Neumann et al. 
Seeber et al.

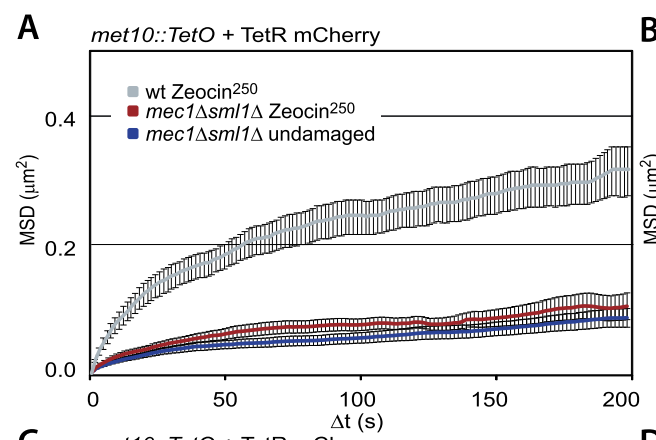

C met10::Teto + TetR mCherry
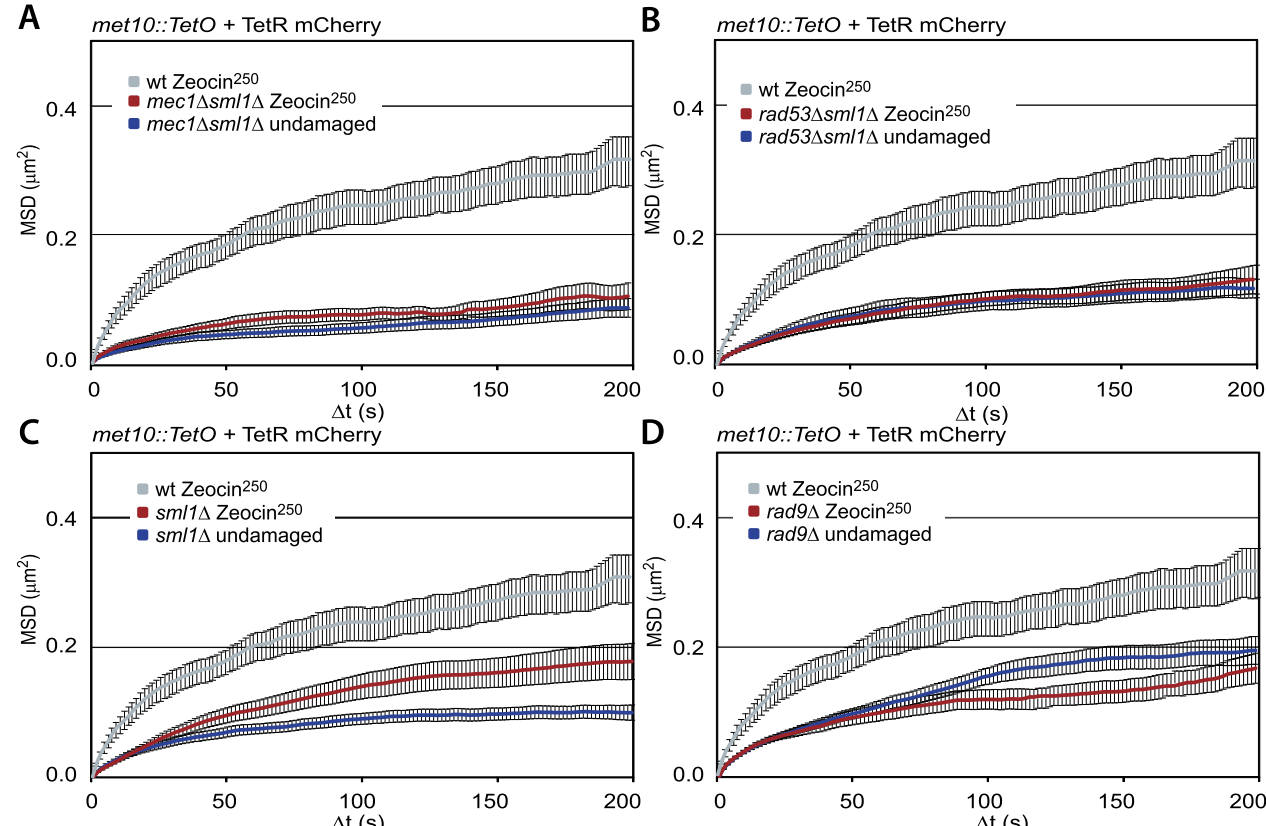

D met10::TetO + TetR mCherry

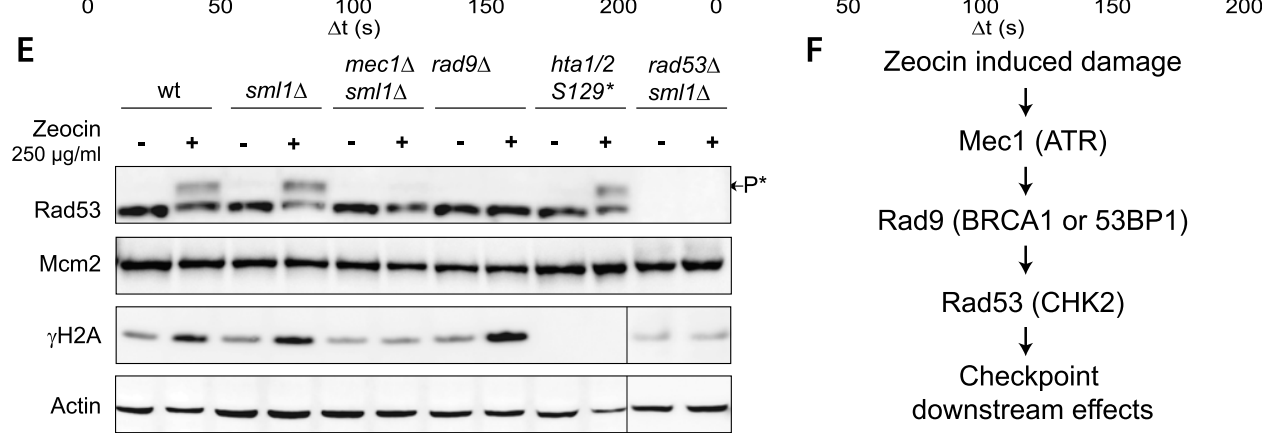

Figure 2. Checkpoint proteins Mec1, Rad9, and Rad53 are essential for damage-induced increases in global chromatin mobility. $(A-D)$ MSD of met $10::$ TetO in S-phase wild-type GA-6879 after $1 \mathrm{~h}$ of $250 \mu \mathrm{g} / \mathrm{mL}$ Zeocin (gray). MSD plots of the same locus without damage

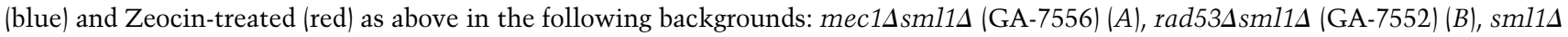
(GA-7553) (C), and rad9s (GA-7555) (D). The error bars show the SEM. The numbers of movies tracked and parameters are given in Table 1. (E) Western blots showing checkpoint activation after $1 \mathrm{~h}$ of Zeocin $(250 \mu \mathrm{g} / \mathrm{mL})$ as in Figure 1B, in wild-type (wt) (GA-6879),

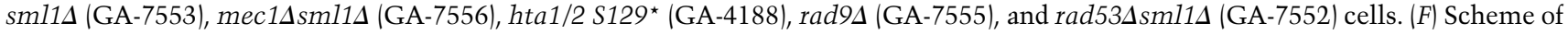
key kinases and regulators in the DNA damage checkpoint.

2012) and has recently been implicated in various DSB repair events (Agmon et al. 2013), we hypothesized that it may have a role in increasing global chromatin mobility. We found that strains lacking either Arp5 or Arp8 abolish increased chromatin mobility after Zeocin treatment (Fig. 4A,B). Complementation of arp $8 \Delta$ with wild-type Arp8 under its endogenous promoter restores chromatin mobility after Zeocin-induced damage just as it restores growth on Zeocin-containing plates (Fig. 4B,C). Importantly, we show that arp $8 \Delta$ does not impair checkpoint activation on Zeocin (Fig. 4D), confirming previously published results on hydroxyurea (van Attikum et al. 2004). In contrast to INO80, we found that the Chd1 and Swrl chromatin remodelers do not have an effect on global chromatin mobility, nor does the sister chromatid cohesion-promoting factor Tof1 (Supplemental Fig. S4; Supplemental Table S2).

To confirm that the INO80 complex is needed for global chromatin mobility in direct response to Mec1 activation, we targeted Ddc1/Ddc2 to activate movement in the absence of damage. Under these conditions, Arp8 was partially required to increase the mobility of a locus in cis (analogous to the partial effect of arp $8 \Delta$ on DSB mobility) (Neumann et al. 2012), and its loss completely compromised the increased mobility of an undamaged locus (LEU2) (Fig. 4E,F). We conclude that the INO80 complex acts downstream from checkpoint activation and is needed to increase global chromatin mobility even when the checkpoint is activated artificially, without widespread DNA damage (Bonilla et al. 2008). The model in Figure 5 illustrates the two pathways that lead to enhanced chromatin mobility-one acting locally, and the second affecting chromatin mobility globally-both showing dependence on INO80.

\section{Discussion}

This study resolves the discrepancy between previous studies (Dion et al. 2012; Mine-Hattab and Rothstein 


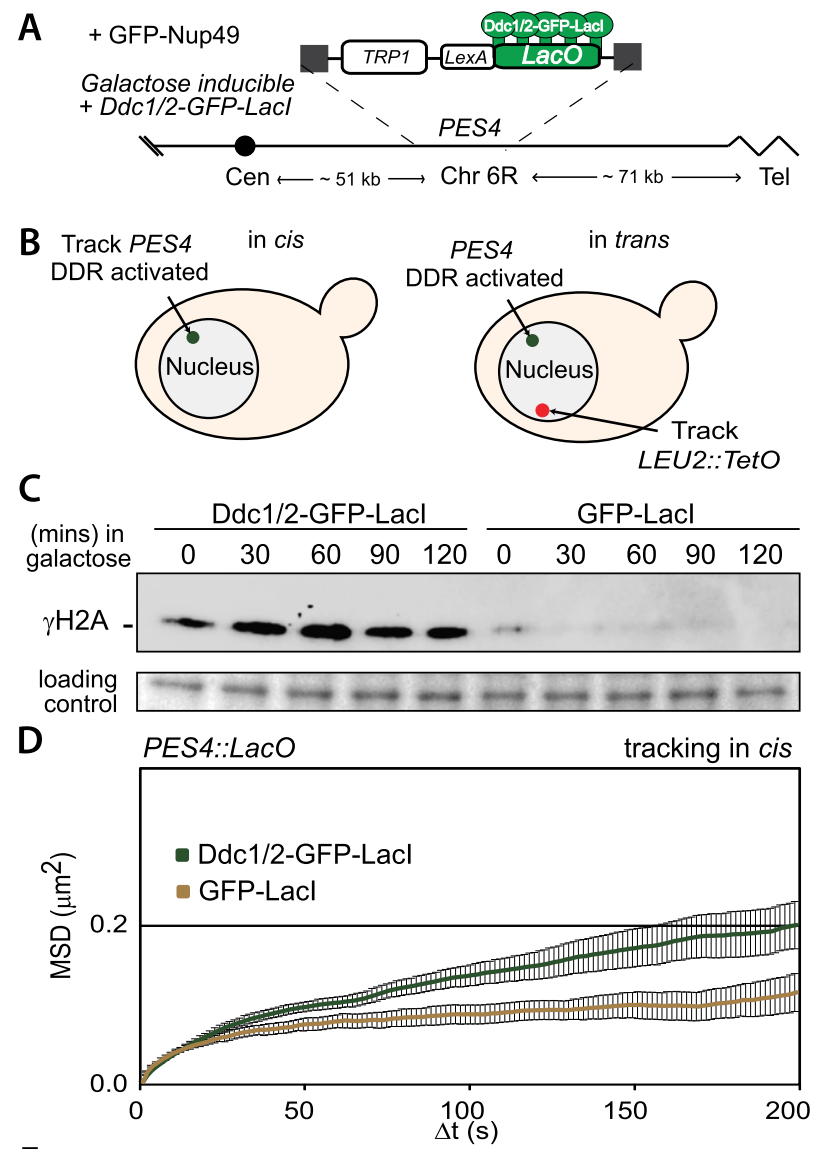

E

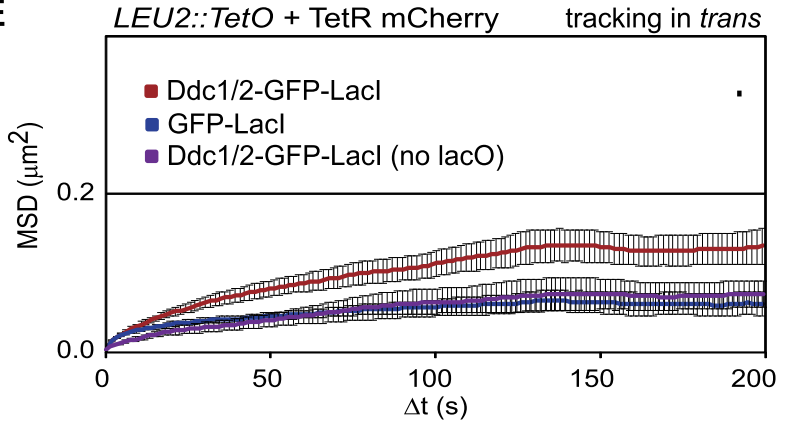

Figure 3. Checkpoint activation is sufficient to enhance both local and global chromatin mobility. (A) Schematic of strain GA7676 showing the tracked locus at PES4. (B) Cartoon illustrating in cis tracking of Ddc1/Ddc2-GFP-LacI foci at PES4 or in trans tracking of mCh-TetO at LEU2. (C) Western blot for H2A S129 phosphorylation after galactose induction of pGAL-Ddc1/2-GFPLacI in GA-7676 or a wild-type (wt) strain, GA-1461. (D) MSD plots of LacO-tagged PES4 during $\mathrm{S}$ phase after $1 \mathrm{~h}$ on galactose in cells expressing Ddc1/Ddc2-GFP-LacI (green; GA-7676) or GFP-LacI (brown; GA-1461). (E) TetR-mCherry at LEU2 in a strain containing GFP-LacI (blue; GA-8088), expressing Ddc1/ Ddc2-GFP-LacI in the presence (red; GA-8023) or absence (purple; GA-8158) of PES4::1acO. The numbers of movies tracked and parameters are given in Table 1.

2012) with respect to chromatin mobility at undamaged loci in cells exposed to damage. Chromatin mobility does indeed increase globally, yet the increase appears to require a threshold level of damage, which correlates with the induction of the DDR through Mec1 and Rad53 kinases. Low-level damage (e.g., after incubation with $50 \mu \mathrm{g} / \mathrm{mL}$ Zeocin) does not provoke a detectable increase in general chromatin movement. We rule out other explanations for the discrepancy, such as cell ploidy or the type of damage induced. Moreover, we can exclude that the global increase in movement arises from chromosome fragmentation given that we scored a checkpoint kinase-dependent increase in mobility in the absence of damage. Finally, by scoring multiple loci, including telomeres, we rule out that the effect depends on the chromosomal context of the locus monitored.

Our studies suggest that changes in chromatin structure that lead to increased mobility in response to DNA damage are different at the site of damage as compared with an undamaged locus. Enhanced mobility in cis requires the repair factors $\operatorname{Rad} 51$ and $\operatorname{Rad} 54$ but is independent of Rad53 kinase activation. Global chromatin mobility increases require the downstream checkpoint kinase Rad53 but not the repair protein Rad51. We note that loss of Arp8 has only a partial effect on the increased mobility of a DSB, while it is essential for the global increase (Fig. 4B,D,F).

We speculate that differential control of chromatin mobility at damaged and undamaged sites may be advantageous to the cell. The enhanced movement of a DSB enhances the probability of harmful translocations or deletions even as it promotes a homology search for HR-mediated repair. The movement, like the checkpoint activation itself, is dependent on the level of damage, consistent with the two being linked. Mec1-Ddc2 activation requires both a threshold and a specific processing event at damage, which may be used to determine in which circumstances global chromatin movement should be enhanced to maximize recombinational repair. The genomic tradeoff for movement is likely to be an elevated risk of deleterious recombination (Dion and Gasser 2013; Seeber et al. 2013).

Several observations suggest that our insights are likely to be relevant to mammalian genomes. For one, recurrent translocations in B lymphocytes occur proportionally to DNA damage (Hakim et al. 2012), and down-regulation of 53BP1 (Rad9) reduces both chromatin mobility and chromosomal end-to-end fusions (Dimitrova et al. 2008). Furthermore, the mobility of arrays that generate translocations in mammalian cells is significantly higher than that of arrays not producing translocations (Roukos et al. 2013). We propose that lesions that do not require a long-range search for a homologous donor and those that do not activate a checkpoint response also fail to trigger a general increase in chromatin mobility.

In summary, we show here that a DNA damagetriggered kinase response controls chromatin organization, with the likely effector being the INO80 complex (Fig. 5). We monitor this as expanded Rc values for undamaged fluorescently tagged chromatin loci. This may reflect local changes in chromatin structure (Neumann et al. 2012) or alterations in the long-range folding of chromatin genome-wide. When S-phase damage is repaired from the sister chromatid, damage movement is 
Seeber et al.

A

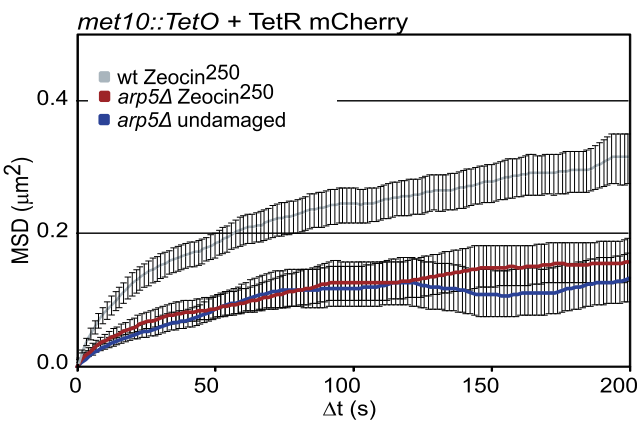

C

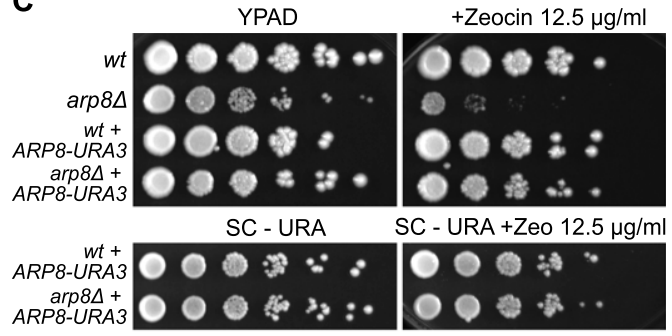

D
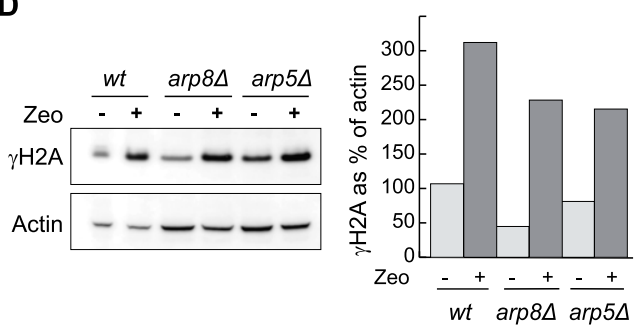

B

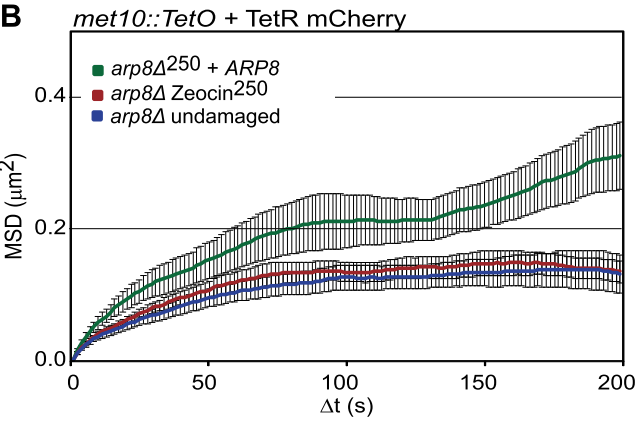

E

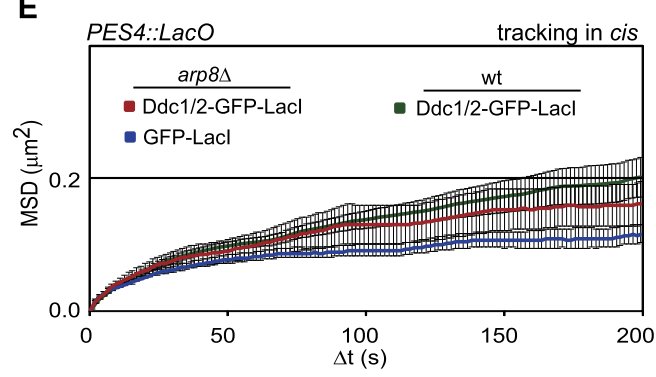

F

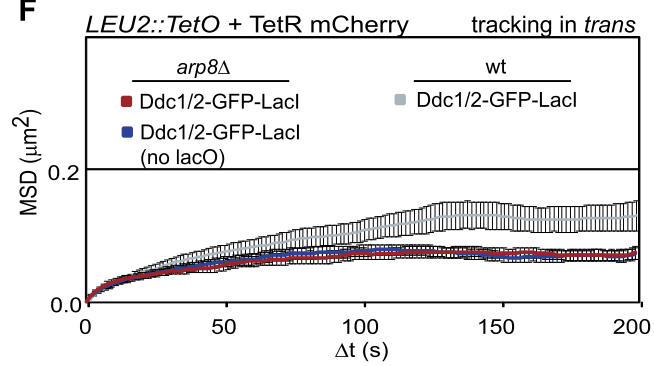

Figure 4. The INO80 complex is required to increase global chromatin mobility. MSD plots of met10::TetO during S phase in wildtype cells after $1 \mathrm{~h}$ in $250 \mu \mathrm{g} / \mathrm{mL}$ Zeocin (gray) and of the same locus without damage (blue) and treated with Zeocin $250 \mu \mathrm{g} / \mathrm{mL}$ (red) in the following mutant backgrounds: arp5s (GA-8202) (A) and arp8s (GA-8132) (B). (C) Serial dilution (10×) showing complementation of arp8s with p416-ARP8-URA3 (Shen et al. 2003). (D) Western blot of $\gamma \mathrm{H} 2 \mathrm{~A}$ accumulation after Zeocin treatment. (E) MSD plot of LacOtagged PES4 during S phase after $1 \mathrm{~h}$ on galactose in cells expressing Ddc1/Ddc2-GFP-LacI (red; GA-8203) or GFP-LacI (blue; GA-8204) in arp8s versus wild-type (green) backgrounds. (F) TetR-mCherry at LEU2 in a strain expressing Ddc1/Ddc2-GFP-LacI (red; GA-8203) or expressing Ddc1/Ddc2-GFP-LacI in the absence of PES4::1acO (blue; GA-8204) versus wild-type (gray). The error bars in all panels show the SEM. The numbers of movies tracked and parameters are given in Table 1.

constrained and not enhanced, which can be overcome by destruction of cohesin (Dion et al. 2013). Thus, we speculate that the checkpoint kinases Mec1-Ddc2 and Rad53 modify INO80 and possibly cohesin in response to damage to regulate global chromatin mobility differentially during the DDR.

\section{Materials and methods}

\section{Yeast growth conditions and plasmids}

Yeast strains used in this study were W303-derived (see Supplemental Table S1). Yeast growth was at $30^{\circ} \mathrm{C}$, and imaging was at $25^{\circ} \mathrm{C}$. Zeocin exposure experiments were done in synthetic complete (SC) medium with 1-h incubations with drugs prior to microscopy or other assays, which were performed in fresh SC medium. Precise conditions are in the Supplemental Material. $p G A L-E C O R 1$ plasmid was a gift of Dr. P. Schär, and the pseudodiploid strain was constructed by integrating a MAT $\boldsymbol{\alpha}$ plasmid at URA3 (gift of Dr. S. Marcand).

\section{Microscopy, movie analysis, and zoning assay}

Live microscopy used an Olympus IX81 microscope equipped with a Yokogawa CSU-X1 scan head, an EM-CCD Cascade II (Photometrics), an ASI MS-2000 Z-piezo stage, and a PlanApo $\times 100$, NA 1.45 total internal reflection fluorescence microscope oil objective. For excitation and exposure times, see the Supplemental Material.

Time-lapse image stacks were analyzed as in Dion et al. (2012) using a custom-made Fiji plug-in (Sage et al. 2005). Analysis of locus position was performed with the zoning assay described in Meister et al. (2010), and phototoxicity was tested by exposing wild-type cells (GA-6879) to standard imaging conditions and then following outgrowth for $5 \mathrm{~h}$ by morphological analysis, comparing them with unexposed cells.

\section{Western blotting}

DDR activation was scored by Western blotting TCA-precipitated proteins separated on a SDS-PAGE gel (Invitrogen). The antibodies used are noted in the Supplemental Material. 


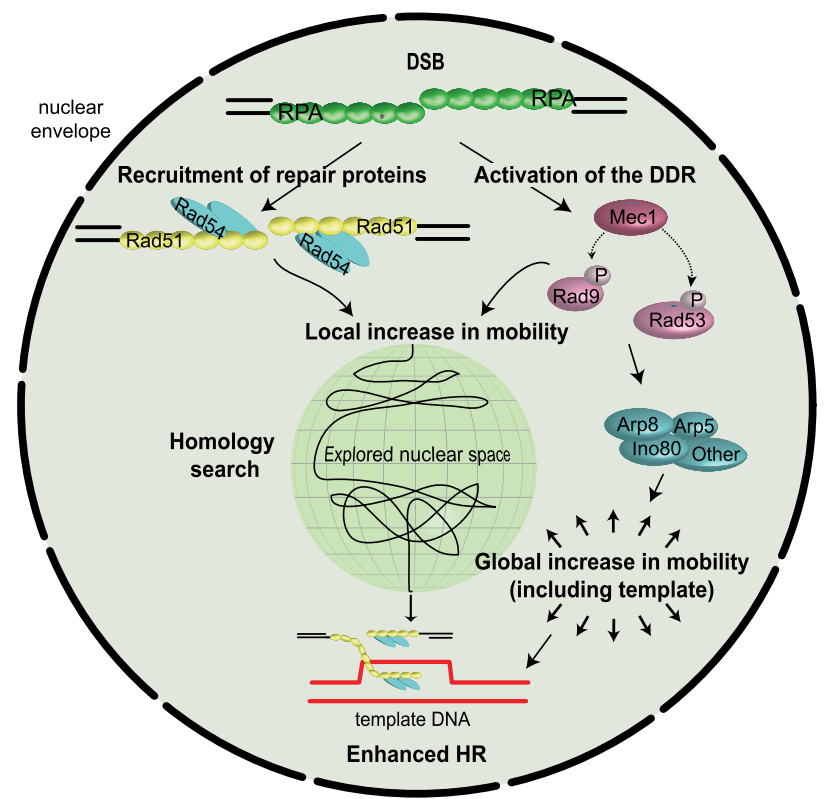

Figure 5. Model of the factors influencing local and global chromatin mobility in response to a DSB. Resection at a DSB leads to accumulation of ssDNA and binding of RPA. This signals the recruitment of repair proteins such as Rad51, Rad52, and finally, Rad54 and activates the DDR. At a DSB, Rad51, Rad54, Rad9, and Mec1 are required to increase DSB mobility (Dion et al. 2012). Global chromatin movement is enhanced by the checkpoint kinase activation and requires Rad53. Downstream from checkpoint activation, the INO80 subunits Arp5 and Arp8 are required to increase mobility globally and have a partial effect on the increase scored at a DSB. Increased mobility of both damaged and undamaged loci would facilitate contact between the DSB and ectopic sites of homology for HR.

\section{Acknowledgments}

We thank C. Horigome, U. Rass, I. Marcomini, P. Zeller, M. Hauer, and M. Di Pietro for critical reading and preparation of the manuscript and figures; P. Schär, S. Marcand, and D.P. Toczyski for reagents; and the Friedrich Miescher Institute Facility for Advanced Imaging and Microscopy for technical assistance. The Gasser laboratory is supported by FP7 Marie Curie Network Nucleosome 4D, the Swiss National Science Foundation National Centre for Competence in Research, "Frontiers in Genetics," and the Friedrich Miescher Institute for Biomedical Research. A.S., V.D., and S.M.G. planned experiments and wrote the paper, and A.S. and V.D. performed experiments.

\section{References}

Agmon N, Liefshitz B, Zimmer C, Fabre E, Kupiec M. 2013. Effect of nuclear architecture on the efficiency of doublestrand break repair. Nat Cell Biol 15: 694-699.

Bonilla CY, Melo JA, Toczyski DP. 2008. Colocalization of sensors is sufficient to activate the DNA damage checkpoint in the absence of damage. Mol Cell 30: 267-276.

Burger RM. 1998. Cleavage of nucleic acids by bleomycin. Chem Rev 98: 1153-1170.

Bystricky K, Van Attikum H, Montiel MD, Dion V, Gehlen L, Gasser SM. 2009. Regulation of nuclear positioning and dynamics of the silent mating type loci by the yeast $\mathrm{Ku} 70$ / Ku80 complex. Mol Cell Biol 29: 835-848.
Dimitrova N, Chen YC, Spector DL, de Lange T. 2008. 53BP1 promotes non-homologous end joining of telomeres by increasing chromatin mobility. Nature 456: 524-528.

Dion V, Gasser SM. 2013. Chromatin movement in the maintenance of genome stability. Cell 152: 1355-1364.

Dion V, Kalck V, Horigome C, Towbin BD, Gasser SM. 2012. Increased mobility of double-strand breaks requires $\mathrm{Mec1}$, Rad9 and the homologous recombination machinery. Nat Cell Biol 14: 502-509.

Dion V, Seeber A, Gasser SM. 2013. Cohesin and the nucleolus constrain the mobility of spontaneous repair foci. EMBO Rep (in press).

Forget AL, Kowalczykowski SC. 2012. Single-molecule imaging of DNA pairing by RecA reveals a three-dimensional homology search. Nature 482: 423-427.

Gartenberg MR, Neumann FR, Laroche T, Blaszczyk M, Gasser SM. 2004. Sir-mediated repression can occur independently of chromosomal and subnuclear contexts. Cell 119: 955-967.

Gehlen L, Gasser SM, Dion V. 2011. How broken DNA finds its template for repair: A computational approach. Prog Theor Phys 191: 20-29.

Haber JE. 2012. Mating-type genes and MAT switching in Saccharomyces cerevisiae. Genetics 191: 33-64.

Hakim O, Resch W, Yamane A, Klein I, Kieffer-Kwon KR, Jankovic M, Oliveira T, Bothmer A, Voss TC, AnsarahSobrinho C, et al. 2012. DNA damage defines sites of recurrent chromosomal translocations in B lymphocytes. Nature 484: 69-74.

Heun P, Laroche T, Shimada K, Furrer P, Gasser SM. 2001. Chromosome dynamics in the yeast interphase nucleus. Science 294: 2181-2186.

Ira G, Hastings PJ. 2012. DNA breakage drives nuclear search. Nat Cell Biol 14: 448-450.

Krawczyk PM, Borovski T, Stap J, Cijsouw T, Cate RT, Medema JP, Kanaar R, Franken NA, Aten JA. 2012. Chromatin mobility is increased at sites of DNA double-strand breaks. J Cell Sci 125: 2127-2133.

Lisby M, Barlow JH, Burgess RC, Rothstein R. 2004. Choreography of the DNA damage response: Spatiotemporal relationships among checkpoint and repair proteins. Cell 118: 699713.

Martin SG, Laroche T, Suka N, Grunstein M, Gasser SM. 1999. Relocalization of telomeric $\mathrm{Ku}$ and SIR proteins in response to DNA strand breaks in yeast. Cell 97: 621-633.

Meister P, Gehlen LR, Varela E, Kalck V, Gasser SM. 2010. Visualizing yeast chromosomes and nuclear architecture. Methods Enzymol 470: 535-567.

Melo JA, Cohen J, Toczyski DP. 2001. Two checkpoint complexes are independently recruited to sites of DNA damage in vivo. Genes Dev 15: 2809-2821.

Mine-Hattab J, Rothstein R. 2012. Increased chromosome mobility facilitates homology search during recombination. Nat Cell Biol 14: 510-517.

Morgan EA, Shah N, Symington LS. 2002. The requirement for ATP hydrolysis by $S$. cerevisiae Rad51 is bypassed by matingtype heterozygosity or RAD54 in high copy. Mol Cell Biol 22: 6336-6343.

Morrison AJ, Kim JA, Person MD, Highland J, Xiao J, Wehr TS, Hensley S, Bao Y, Shen J, Collins SR, et al. 2007. Mec1/Tel1 phosphorylation of the INO80 chromatin remodeling complex influences DNA damage checkpoint responses. Cell 130: 499-511.

Neumann FR, Dion V, Gehlen LR, Tsai-Pflugfelder M, Schmid R, Taddei A, Gasser SM. 2012. Targeted INO80 enhances subnuclear chromatin movement and ectopic homologous recombination. Genes Dev 26: 369-383. 
Seeber et al.

Ponti A, Schwarb P, Gulati A, Bäcker V. 2007. Huygens remote manager. Imag Microscopy 9: 57-58.

Povirk LF. 1996. DNA damage and mutagenesis by radiomimetic DNA-cleaving agents: Bleomycin, neocarzinostatin and other enediynes. Mutat Res 355: 71-89.

Renkawitz J, Lademann CA, Kalocsay M, Jentsch S. 2013. Monitoring homology search during DNA double-strand break repair in vivo. Mol Cell. 50: 261-272.

Roukos V, Voss TC, Schmidt CK, Lee S, Wangsa D, Misteli T. 2013. Spatial dynamics of chromosome translocations in living cells. Science 341: 660-664.

Sage D, Neumann FR, Hediger F, Gasser SM, Unser M. 2005. Automatic tracking of individual fluorescence particles: Application to the study of chromosome dynamics. IEEE Trans Image Process 14: 1372-1383.

Seeber A, Hauer M, Gasser SM. 2013. Nucleosome remodelers in double-strand break repair. Curr Opin Genet Dev 23: 174184.

Shen X, Ranallo R, Choi E, Wu C. 2003. Involvement of actinrelated proteins in ATP-dependent chromatin remodeling. Mol Cell 12: 147-155.

Smolka MB, Albuquerque CP, Chen SH, Zhou H. 2007. Proteome-wide identification of in vivo targets of DNA damage checkpoint kinases. Proc Natl Acad Sci 104: 10364-10369.

van Attikum H, Fritsch O, Hohn B, Gasser SM. 2004. Recruitment of the INO80 complex by H2A phosphorylation links ATP-dependent chromatin remodeling with DNA double-strand break repair. Cell 119: 777-788.

Vialard JE, Gilbert CS, Green CM, Lowndes NF. 1998. The budding yeast Rad9 checkpoint protein is subjected to Mec1/ Tell-dependent hyperphosphorylation and interacts with Rad53 after DNA damage. EMBO J 17: 5679-5688.

Weber SC, Spakowitz AJ, Theriot JA. 2012. Nonthermal ATPdependent fluctuations contribute to the in vivo motion of chromosomal loci. Proc Natl Acad Sci 109: 7338-7343.

Wilson JH, Leung WY, Bosco G, Dieu D, Haber JE. 1994. The frequency of gene targeting in yeast depends on the number of target copies. Proc Natl Acad Sci 91: 177-181.

Zhao X, Muller EG, Rothstein R. 1998. A suppressor of two essential checkpoint genes identifies a novel protein that negatively affects dNTP pools. Mol Cell 2: 329-340. 


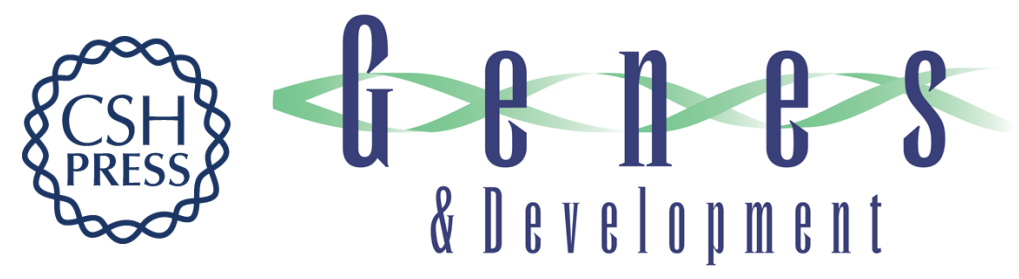

\section{Checkpoint kinases and the INO80 nucleosome remodeling complex enhance global chromatin mobility in response to DNA damage}

Andrew Seeber, Vincent Dion and Susan M. Gasser

Genes Dev. 2013, 27: originally published online September 12, 2013

Access the most recent version at doi:10.1101/gad.222992.113

\section{Supplemental http://genesdev.cshlp.org/content/suppl/2013/09/09/gad.222992.113.DC1 Material}

References This article cites 36 articles, 12 of which can be accessed free at: http://genesdev.cshlp.org/content/27/18/1999.full.html\#ref-list-1

Creative This article is distributed exclusively by Cold Spring Harbor Laboratory Press for the first Commons six months after the full-issue publication date (see

License http://genesdev.cshlp.org/site/misc/terms.xhtml). After six months, it is available under a Creative Commons License (Attribution-NonCommercial 3.0 Unported), as described at http://creativecommons.org/licenses/by-nc/3.0/.

Email Alerting Receive free email alerts when new articles cite this article - sign up in the box at the top Service right corner of the article or click here.

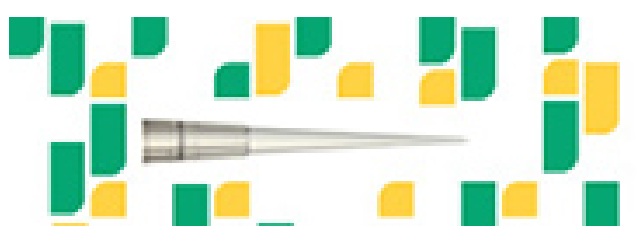

Focused on your science. 